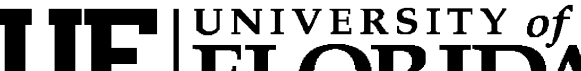 FLORIDA \\ IFAS Extension
}

\section{Weed Control in Cucurbit Crops (Muskmelon, Cucumber, Squash, and Watermelon) ${ }^{1}$}

William M. Stall ${ }^{2}$

\section{Crop Competition}

An often overlooked tool to reduce weed competition is to establish a good crop stand in which plants emerge and rapidly shade the ground. The plant that emerges first and grows the most rapidly is the plant that will have the competitive advantage. Utilization of good production management practices such as fertility, well-adapted varieties, proper water control (irrigation, drainage), and establishment of adequate plant populations is very helpful in reducing weed competition. Everything possible should be done to ensure that the crop, not the weeds, has the competitive advantage. Tests with watermelons and muskmelons have shown that if weeds such as smooth pigweed emerge 4 to 5 weeks after the crops, they will not reduce yield of the crop. If the weed emerges and competes with the crop in the first 4 weeks, however, yield will be reduced by competition. Two nightshade plants growing in-row between watermelon plants have been shown to reduce yield $80-100 \%$ in open culture and $60-75 \%$ in mulch culture production.

\section{Mechanical Control}

Mechanical control includes field preparation by plowing or disking, cultivation, mowing, hoeing and hand pulling of weeds. Mechanical control practices are among the oldest of weed management techniques. Seedbed preparation by plowing or disking exposes many weed seeds to variations in light, temperature, and moisture. For some weeds, this process breaks weed-seed dormancy, leading to early season control with herbicides or additional cultivation.

Cultivate only deeply enough in the row to achieve weed control; deep cultivation may prune roots, bring weed seeds to the surface, and disturb soil previously treated with a herbicide. Follow the same precautions between rows. The roots of watermelons may extend as far as the tips of the vines, even when grown on mulch. Turning the vines and deep cultivation in the vine area may destroy a large number of roots, reducing water and nutrient uptake. Cultivation in a timely fashion is extremely important.

1. This document is HS190, one of a series of the Horticultural Sciences Department, Florida Cooperative Extension Service, Institute of Food and Agricultural Sciences, University of Florida. Original publication date March 1, 1999. Revised April 2009. Visit the EDIS Web Site at http://edis.ifas.ufl.edu.

2. William M. Stall, professor, Horticultural Sciences Department, Cooperative Extension Service, Institute of Food and Agricultural Sciences, University of Florida, Gainesville, 32611.

The use of trade names in this publication is solely for the purpose of providing specific information. It is not a guarantee or warranty of the products named, and does not signify that they are approved to the exclusion of others of suitable composition. 


\section{Mulching}

The use of polyethylene mulch has been shown many times to increase yield and earliness of cucurbits. The proper injection of fumigants under the mulch will control nematodes, soil insects, soil-borne diseases and weed seeds. Mulches act as a barrier to the growth of many weeds. Nutsedges, however, are weeds that can and will grow through the mulch.

\section{Herbicides}

Properly selected herbicides are effective tools for weed control in cucurbits. Most of the labeled herbicides are for preplant or preemergence applications to the crop and weeds. Only two herbicides are labeled at the present time for postemergent crop applications. Care must be exercised to use these materials at the proper rate and correct time to avoid crop damage. Cucurbits as a group have very limited tolerances to most herbicides.

Two herbicides were removed for use on cucurbit crops by their respective companies in 1985 due to crop injuries resulting from "inadvertent misuse." This severely limits the number of herbicides available for use in Florida.

Before applying a herbicide, carefully calibrate your sprayer. Make sure the proper speed, pressure and nozzles are being used in the field. Worn nozzles can increase gallonage sprayed significantly. Always use the same size nozzles.

Most of the new herbicides being tested for labeling on cucurbits have a narrow range of tolerance. A mistake in calibration or application will cause damage to the crop. They must also be applied in the proper manner.

There are 2 categories of soil applied herbicides: surface-applied and incorporated herbicides. Surface-applied herbicides require rainfall or irrigation shortly after application for best results. Lack of moisture often results in poor weed control, however, they are relatively easy to apply.
Incorporated herbicides are not dependent on rainfall or irrigation and have generally given more consistent and wider-spectrum control. They do, however, require more time and equipment for incorporation.

Herbicides labeled for surface application may cause phytotoxicity to melons if incorporated, however.

Do not use herbicides that are not labeled for use in Florida. Use of unregistered materials can result in destruction of the crop, a fine or both. Use of herbicides with pending labels can also delay or jeopardize subsequent registrations.

The EPA has recently defined for tolerance purposes what crops may be included under certain general commodity names. The general term "melon" on a label includes the specific commodities: muskmelons, including hybrids and/or varieties of Cucumis melo (including true cantaloupe, cantaloupe, casaba, Santa Claus melon, crenshaw melon, honeydew melon, honey balls, Persian melon, golden pershaw melon, mango melon, pineapple melon, snake melon); and watermelons, including hybrids and/or varieties of Citrullus spp.

The term "Summer squash" includes fruits of the gourd (Cucurbitaceae) family. Fruits in this category are consumed when immature, are $100 \%$ edible either cooked or raw, cannot be stored once picked, have a soft rind which is easily penetrated, and have seeds which, if they were harvested, would not germinate; e.g. Cucurbita pepo (Ie. crookneck squash, straightneck squash, scallop squash, and vegetable marrow); Laginaria spp (Ie. spaghetti squash, hyotan, cucuzza); Luffa spp. (Ie. hechima, Chinese okra); Memordica spp. (Ie. bitter melon, balsam pear, balsam apple, Chinese cucumber); and other varieties and/or hybrids of these.

Herbicides must be applied at exactly the correct rate and time to selectively control weed growth in a vegetable crop. Obtain consistent results by reading the herbicide label and other information about the proper application and timing of each herbicide. To avoid confusion between commercial formulations, suggested rates listed in Table 1 are stated as pounds 
active ingredient per acre (lbs, ai./acre). Read and follow all label directions. 
Table 1. Chemical weed controls: cucurbit crops (muskmelons, cucumbers, squash, watermelon).

\begin{tabular}{|c|c|c|c|c|}
\hline \multirow[t]{2}{*}{ Herbicide } & \multirow[t]{2}{*}{ Labeled Crops } & \multirow{2}{*}{$\begin{array}{l}\text { Time of Application } \\
\text { to Crop }\end{array}$} & \multicolumn{2}{|c|}{ Rate (Ibs. ai./acre) } \\
\hline & & & Mineral & Muck \\
\hline $\begin{array}{l}\text { Bensulide } \\
\text { (Prefar 4E) }\end{array}$ & $\begin{array}{l}\text { Cucurbit vegetable group: } \\
\text { cucumbers, melons, squash } \\
\text { (summer and winter), pumpkins, } \\
\text { edible gourds, bitter melon }\end{array}$ & $\begin{array}{l}\text { Preplant incorporated, } \\
\text { Preemergence }\end{array}$ & $5.0-6.0$ & --- \\
\hline \multicolumn{5}{|c|}{$\begin{array}{l}\text { Remarks: Controls germinating grasses. Incorporate } 1 \text { to } 2 \text { inches. Note precautions about reapplying within } 12 \\
\text { months and planting non-registered crops within } 18 \text { months. Label states control of crabgrass, foxtail, goosegrass, fall } \\
\text { panicum and sprangletop. }\end{array}$} \\
\hline $\begin{array}{l}\text { Bensulide + Naptalam } \\
\text { (Prefar 4E + Alanap) }\end{array}$ & $\begin{array}{l}\text { Cantaloupes, muskmelons, } \\
\text { cucumbers, watermelons }\end{array}$ & Preplant or Preemergence & $\begin{array}{l}5.0 \text { (Bensulide) }+ \\
3.0-4.0 \\
\text { (Naptalam) }\end{array}$ & ---- \\
\hline \multicolumn{5}{|c|}{$\begin{array}{l}\text { Remarks: Combination (tank mix) will provide wider range of weed control than either material alone. Incorporate into } \\
\text { the soil lightly ( } 0.5 \text { to } 1.0 \text { inch) with suitable equipment prior to planting or incorporate preemergent treatments with } \\
\text { overhead irrigation. Follow all precautions on both labels. }\end{array}$} \\
\hline $\begin{array}{l}\text { Carfentrazone } \\
\text { (Aim) }\end{array}$ & $\begin{array}{l}\text { Cucurbit crop group } \\
\text { (all) }\end{array}$ & $\begin{array}{l}\text { Preplant } \\
\text { Directed-hooded } \\
\text { Row-middles } \\
\end{array}$ & 0.031 & 0.031 \\
\hline \multicolumn{5}{|c|}{$\begin{array}{l}\text { Remarks: Aim may be applied as a preplant burndown treatment and/or as a post-directed hooded application to row } \\
\text { middles for the burndown of emerged broadleaf weeds. May be tank mixed with other registered herbicides. May be } \\
\text { applied at up to } 2 \text { oz ( } 0.031 \mathrm{lb} \text { ai). Use a quality spray adjuvant such as crop oil concentrate (coc) or non-ionic surfactant } \\
\text { at recommended rates. }\end{array}$} \\
\hline $\begin{array}{l}\text { Clethodim } \\
\text { (Select) } \\
\text { (Arrow) } \\
\text { (Select Max) } \\
\end{array}$ & $\begin{array}{l}\text { Cucurbits (cucumber, squash, } \\
\text { melons and all commodities in } \\
\text { crop group) }\end{array}$ & Postemergence & $0.1-0.125$ & --- \\
\hline \multicolumn{5}{|c|}{$\begin{array}{l}\text { Remarks: Use Select for the control of annual and perennial grasses. Use a crop-oil concentrate at } 1 \% \mathrm{v} / \mathrm{v} \text { in the } \\
\text { finished spray volume. Do not apply more than } 8 \mathrm{fl} \text {. oz. product/A per application. Do not apply within } 14 \text { days of } \\
\text { harvest. Rate for Select Max is } 9-16 \text { floz/A with the use of a non-ionic surfactant. }\end{array}$} \\
\hline $\begin{array}{l}\text { Clomazone } \\
\text { (Command } 3 \mathrm{ME} \text { ) }\end{array}$ & $\begin{array}{l}\text { Summer squash } \\
\text { Winter squash }\end{array}$ & $\begin{array}{l}\text { Preemergence } \\
\text { Preemergence } \\
\text { Row Middles }\end{array}$ & $\begin{array}{l}0.15 \\
0.25-0.75 \\
0.75\end{array}$ & --- \\
\hline \multicolumn{5}{|c|}{$\begin{array}{l}\text { Remarks: Labeled rate for summer squash is } 0.25 \mathrm{lb} \text { a.i. Bleaching has been seen under adverse conditions at this } \\
\text { rate. Suggest use as tank mix to increase efficacy. May be applied to winter squash and processing pumpkins. See label } \\
\text { for varieties and cultivars where application is prohibited. Do not use on Jack-O-Lantern type pumpkins. May be used } \\
\text { on processing type varieties. Read disclaimer on the label before use. }\end{array}$} \\
\hline $\begin{array}{l}\text { DCPA } \\
\text { (Dacthal W-75) } \\
\text { (Dacthal 6F) }\end{array}$ & $\begin{array}{l}\text { Seeded melons: cantaloupe, } \\
\text { honeydew, watermelon; } \\
\text { cucumber; squash (summer, } \\
\text { winter) }\end{array}$ & Early postemergence & $6-8$ & ---- \\
\hline \multicolumn{5}{|c|}{$\begin{array}{l}\text { Remarks: Apply only when plants have } 4 \text { to } 5 \text { true leaves and are well established and when growing conditions are } \\
\text { favorable for good plant growth. Does not control emerged weeds. If weeds have emerged, cultivate prior to application. } \\
\text { Do not incorporate. }\end{array}$} \\
\hline $\begin{array}{l}\text { Ethalfluralin + } \\
\text { Clomazone } \\
\text { (Strategy) }\end{array}$ & $\begin{array}{l}\text { Cucumber, melons, } \\
\text { watermelons, squash, pumpkins }\end{array}$ & $\begin{array}{l}\text { Preemergence } \\
\text { Post-directed }\end{array}$ & $2-3$ pints & --- \\
\hline
\end{tabular}


Table 1. Chemical weed controls: cucurbit crops (muskmelons, cucumbers, squash, watermelon).

\begin{tabular}{|c|c|c|c|c|}
\hline \multirow[t]{2}{*}{ Herbicide } & \multirow[t]{2}{*}{ Labeled Crops } & \multirow{2}{*}{$\begin{array}{l}\text { Time of Application } \\
\text { to Crop }\end{array}$} & \multicolumn{2}{|c|}{ Rate (Ibs. ai./acre) } \\
\hline & & & Mineral & Muck \\
\hline \multicolumn{5}{|c|}{$\begin{array}{l}\text { Remarks: Strategy is a premix of ethalfluralin and clomazone at } 1.5+0.5 \mathrm{lbs} / \text { gal. Apply } 2-3 \text { pints product post seeding } \\
\text { to surface prior to crop and weed emergence. Must be applied no later than } 2 \text { days after seeding. Soil incorporate with } \\
\text { overhead irrigation at } 1 / 2 \text { inch or with a rain(s) at no less than } 2 \text { inches within } 5 \text { days. Excessive rains or irrigation may } \\
\text { cause injury. For furrow irrigation where no rainfall is received, a shallow cultivation may be used to activate the } \\
\text { herbicides. Do not apply before transplanting. Do not apply under row covers, hot caps, or polyethylene mulches. May } \\
\text { be applied as a post-directed spray to row middles after crop emergence or transplanting. Do not apply over plants. } \\
\text { The premix controls a large number of grasses and broadleaf weeds. }\end{array}$} \\
\hline $\begin{array}{l}\text { Flumioxazin } \\
\text { (Chateau) }\end{array}$ & $\begin{array}{l}\text { Melon Group } \\
\text { Muskmelon, Watermelon }\end{array}$ & $\begin{array}{l}\text { Directed } \\
\text { Row-middles }\end{array}$ & 0.125 & +-- \\
\hline \multicolumn{5}{|c|}{$\begin{array}{l}\text { Remarks: Chateau may be applied up to } 4 \text { oz product/application to row middles of raised plastic-mulched beds that } \\
\text { are at least } 4 \text { inches higher than the treated row middle and the mulched bed must be a minimum of a } 24 \text {-inch bed } \\
\text { width. Do not apply after crops are transplanted/seeded. All applications must be made with shielded or hooded } \\
\text { equipment. For control of emerged weeds, a burn down herbicide may be tank-mixed. Label is a Third-Party } \\
\text { registration (TPR, Inc.). Use without a signed authorization and waiver of liability is a misuse of the product. }\end{array}$} \\
\hline $\begin{array}{l}\text { Glyphosate } \\
\text { (Roundup, Durango, } \\
\text { Touchdown, } \\
\text { Glyphomax) }\end{array}$ & Cucurbit Crops & $\begin{array}{l}\text { Chemical fallow } \\
\text { Preplant, pre emergence, } \\
\text { Pre transplant }\end{array}$ & $0.3-1.0$ & ---- \\
\hline \multicolumn{5}{|c|}{$\begin{array}{l}\text { Remarks: Roundup, Glyphomax and Touchdown have several formulations. Check the label of each for specific } \\
\text { labeling directions. }\end{array}$} \\
\hline $\begin{array}{l}\text { Halosulfuron } \\
\text { (Sandea) }\end{array}$ & $\begin{array}{l}\text { Cucumber, cantaloupe, } \\
\text { honeydew and crenshaw melons }\end{array}$ & $\begin{array}{l}\text { Preemergence, } \\
\text { Postemergence } \\
\text { Row middles }\end{array}$ & 0.024 & ---- \\
\hline \multicolumn{5}{|c|}{$\begin{array}{l}\text { Remarks: Apply uniformly at } 1 / 2 \text { oz. product with ground equipment in a minimum of } 15 \text { gallons of water per acre. For } \\
\text { postemergence applications, apply after the crop has reached the } 2 \text { true leaf stage, but before flowering. Use a non } \\
\text { ionic surfactant for postemergence applications. May be used for row middle treatments at up to } 1 \text { oz. product. Controls } \\
\text { actively growing nutsedge species best post. Do not apply within } 30 \text { days of harvest for cucumber and } 57 \text { days for the } \\
\text { melon subgroup. }\end{array}$} \\
\hline $\begin{array}{l}\text { Halosulfuron } \\
\text { (Sandea) }\end{array}$ & $\begin{array}{l}\text { Cucurbit vegetables including } \\
\text { watermelon, squash, pumpkins, } \\
\text { gourds, etc. }\end{array}$ & $\begin{array}{l}\text { Preplant } \\
\text { Pre transplant } \\
\text { Row middles } \\
\end{array}$ & $0.024-0.048$ & ---- \\
\hline \multicolumn{5}{|c|}{$\begin{array}{l}\text { Remarks: May be applied as a preplant or pretransplant application to bare ground or mulch grown cucurbit vegetables } \\
\text { at } 1 / 2 \mathrm{oz} \text { product for the suppression of nutsedges and the control of listed broadleaf weeds. May be applied between } \\
\text { rows for the control of emerged nutsedges and labeled broadleaf weeds at } 1 / 2 \text { to } 1 \text { oz product. Use a non ionic } \\
\text { surfactant in the spray mix. Check the label for phi limitations. }\end{array}$} \\
\hline $\begin{array}{l}\text { Halosulfuron } \\
\text { (Sandea) }\end{array}$ & Watermelon & $\begin{array}{l}\text { Preemergence } \\
\text { Pre transplant }\end{array}$ & $0.024-0.036$ & ---- \\
\hline \multicolumn{5}{|c|}{$\begin{array}{l}\text { Remarks: Sandea may be applied preemergence to seeded watermelon on bare ground culture or pre-seeding to } \\
\text { mulch-cultured melons. Pre-transplant applications may be made also to bare ground or mulched production. } \\
\text { Transplanting should be no sooner than } 7 \text { days after application. application may be } 1 / 2 \text { to } 3 / 4 \text { oz product per acre. Use } \\
\text { lighter rates on sandy soils with low organic matter. }\end{array}$} \\
\hline $\begin{array}{l}\text { Halosulfuron } \\
\text { (Sandea) }\end{array}$ & $\begin{array}{l}\text { Pumpkins } \\
\text { Winter Squash }\end{array}$ & $\begin{array}{l}\text { Preemergence } \\
\text { Pre transplant } \\
\text { Post transplant }\end{array}$ & $0.024-0.036$ & ---- \\
\hline \multicolumn{5}{|c|}{$\begin{array}{l}\text { Remarks: Sandea may be applied after seeding, but before soil cracking or pre transplant. transplanting should not be } \\
\text { made sooner than } 7 \text { days after application. May be applied post over-the-top when plants reach the } 4-5 \text { true leaf stage, } \\
\text { but before first female flowers appear. Application rates are } 1 / 2 \text { to } 3 / 4 \text { oz product per acre. }\end{array}$} \\
\hline Naptalam (Alanap-L) & $\begin{array}{l}\text { Cantaloupes, cucumbers, } \\
\text { watermelons }\end{array}$ & $\begin{array}{l}\text { Preemergence, } \\
\text { Preplant }\end{array}$ & $3.0-4.0$ & ---- \\
\hline
\end{tabular}


Table 1. Chemical weed controls: cucurbit crops (muskmelons, cucumbers, squash, watermelon).

\begin{tabular}{|c|c|c|c|c|}
\hline \multirow[t]{2}{*}{ Herbicide } & \multirow[t]{2}{*}{ Labeled Crops } & \multirow{2}{*}{$\begin{array}{l}\text { Time of Application } \\
\text { to Crop }\end{array}$} & \multicolumn{2}{|c|}{ Rate (Ibs. ai./acre) } \\
\hline & & & Mineral & Muck \\
\hline \multicolumn{5}{|c|}{$\begin{array}{l}\text { Remarks: Apply within } 48 \text { hours of seeding. Apply preemergence to weeds and incorporate with overhead irrigation. } \\
\text { Label states control of germinating annuals such as lambsquarter, ragweed, purslane, cocklebur, white mustard, } \\
\text { shepherdspurse, redroot pigweed, hairy galingosa and carpetweed. }\end{array}$} \\
\hline Naptalam (Alanap-L) & $\begin{array}{l}\text { Cantaloupes, cucumbers, } \\
\text { watermelons }\end{array}$ & $\begin{array}{l}\text { Postemergence, } \\
\text { Posttransplant }\end{array}$ & $3.0-4.0$ & ---- \\
\hline \multicolumn{5}{|c|}{$\begin{array}{l}\text { Remarks: Apply } 1 \text { month after planting when vines are starting to run but before weeds have emerged or immediately } \\
\text { after transplanting. Do not use when plants are under stress due to weather conditions. Do not tank mix with crop oil or } \\
\text { adjuvants. Phytotoxicity may occur. }\end{array}$} \\
\hline $\begin{array}{l}\text { Paraquat } \\
\text { (Gramoxone Inteon) } \\
\text { (Firestorm) }\end{array}$ & $\begin{array}{l}\text { Watermelon, squash, pumpkin, } \\
\text { cantaloupe, muskmelon, } \\
\text { cucumber }\end{array}$ & Preplant or Preemergence & $0.63-0.94$ & --- \\
\hline \multicolumn{5}{|c|}{$\begin{array}{l}\text { Remarks: Controls emerged weeds only. Apply prior, during or after planting, but before crop emerges. Use a non-ionic } \\
\text { spreader. }\end{array}$} \\
\hline $\begin{array}{l}\text { Paraquat } \\
\text { (Gramoxone Inteon) }\end{array}$ & Melons & $\begin{array}{l}\text { Postemergence directed } \\
\text { spray }\end{array}$ & $0.48-0.93$ & ---- \\
\hline \multicolumn{5}{|c|}{$\begin{array}{l}\text { Remarks: Controls emerged weeds only. Apply } 1.0 \text { to } 3.0 \text { pts. per sprayed acre with ground equipment, directing spray } \\
\text { between the rows, and use shields to prevent spray contact with the crop plants. Add a non-ionic surfactant at } 8 \text { fl. oz. } \\
\text { per } 100 \text { gals. of spray mix. Do not apply more than } 3 \text { times per season. }\end{array}$} \\
\hline $\begin{array}{l}\text { Pelargonic Acid } \\
\text { (Scythe) }\end{array}$ & $\begin{array}{l}\text { Cucurbits (melons; cucumber, } \\
\text { gourd, pumpkin, squash, } \\
\text { muskmelon and watermelon) }\end{array}$ & $\begin{array}{l}\text { Preplant } \\
\text { Preemergence } \\
\text { Directed-Shielded }\end{array}$ & $3-10 \% \mathrm{v} / \mathrm{v}$ & $3-10 \% \mathrm{v} / \mathrm{v}$ \\
\hline \multicolumn{5}{|c|}{$\begin{array}{l}\text { Remarks: Product is a contact, non-selective, foliar applied herbicide. There is no residual activity. May be tank mixed } \\
\text { with soil residual compounds. Consult the label for rates and other information. }\end{array}$} \\
\hline $\begin{array}{l}\text { S-Metolachlor } \\
\text { (Dual Magnum) }\end{array}$ & Pumpkin & $\begin{array}{l}\text { Inter-row } \\
\text { Inter-hil }\end{array}$ & $0.188-1.26$ & --- \\
\hline \multicolumn{5}{|c|}{$\begin{array}{l}\text { Remarks: Apply before weeds emerge at } 1.0 \text { to } 1.33 \text { pint/A as an inter-row or inter-hill application in pumpkin. Leave } 1 \\
\text { foot of untreated area over the row, or } 6 \text { inches to each side of the planted hill and/or emerged pumpkin foliage } \\
\text { (inter-row or inter-hill means not directly over the planted seed or young pumpkin plants). Do not apply closer than } 30 \\
\text { days before harvest. }\end{array}$} \\
\hline Sethoxydim (Poast) & Cucurbits: all & Postemergence & $0.188-0.28$ & ---- \\
\hline \multicolumn{5}{|c|}{$\begin{array}{l}\text { Remarks: Controls actively growing grass weeds. A total of } 3 \text { pts. product per acre may be applied in one season. Do } \\
\text { not apply within } 14 \text { days of harvest. Apply in } 5 \text { to } 20 \text { gals. of water adding } 2 \text { pts. of crop oil concentrate per acre. } \\
\text { Unsatisfactory results may occur if applied to grasses under stress. Use } 0.188 \mathrm{lb} \text {. ai. ( } 1 \text { pt.) on seedling grasses and up } \\
\text { to } 0.28 \mathrm{lb} \text {. ai. ( } 1.5 \text { pts.) on perennial grasses emerging from rhizomes, etc. Consult label for grass species and growth } \\
\text { stage for best control. }\end{array}$} \\
\hline Terbacil (Sinbar) & Watermelon & $\begin{array}{l}\text { Preemergence } \\
\text { Pretransplant } \\
\text { Row Middles }\end{array}$ & $0.1-0.2$ & ---- \\
\hline \multicolumn{5}{|c|}{$\begin{array}{l}\text { Remarks: For watermelon only. Apply } 2 \text { to } 4 \text { oz product }(0.1-0.2 \mathrm{lb} \text { ai) to seeded or transplanted watermelon, } \\
\text { preemergence after planting to seeded and pretransplanting for transplanted watermelon. May be applied under mulch } \\
\text { and to row middles. Controls many annual broadleaf weeds. }\end{array}$} \\
\hline
\end{tabular}

